\title{
Design and validation of a pericentromeric BAC clone set aimed at improving diagnosis and phenotype prediction of supernumerary marker chromosomes
}

Chiara Castronovo ${ }^{1 *}$, Emanuele Valtorta ${ }^{1}$, Milena Crippa ${ }^{1}$, Sara Tedoldi ${ }^{2}$, Lorenza Romitti ${ }^{3}$, Maria Cristina Amione ${ }^{4}$, Silvana Guerneri ${ }^{5}$, Daniela Rusconi ${ }^{1}$, Lucia Ballarati ${ }^{1}$, Donatella Milani ${ }^{6}$, Enrico Grosso ${ }^{4}$, Pietro Cavalli ${ }^{2}$, Daniela Giardino ${ }^{1}$, Maria Teresa Bonati ${ }^{7}$, Lidia Larizza ${ }^{1,8}$ and Palma Finelli ${ }^{1,9}$

\begin{abstract}
Background: Small supernumerary marker chromosomes (SSMCs) are additional, structurally abnormal chromosomes, generally smaller than chromosome 20 of the same metaphase spread. Due to their small size, they are difficult to characterize by conventional cytogenetics alone. In regard to their clinical effects, SSMCs are a heterogeneous group: in particular, sSMCs containing pericentromeric euchromatin are likely to be associated with abnormal outcomes, although exceptions have been reported. To improve characterization of the genetic content of sSMCs, several approaches might be applied based on different molecular and molecular-cytogenetic assays, e.g., fluorescent in situ hybridization (FISH), array-based comparative genomic hybridization (array $(G H)$ ), and multiplex ligation-dependent probe amplification (MLPA).

To provide a complementary tool for the characterization of SSMCs, we constructed and validated a new, FISH-based, pericentromeric Bacterial Artificial Chromosome (BAC) clone set that with a high resolution spans the most proximal euchromatic sequences of all human chromosome arms, excluding the acrocentric short arms.

Results: By FISH analysis, we assayed 561 pericentromeric BAC probes and excluded 75 that showed a wrong chromosomal localization. The remaining 486 probes were used to establish 43 BAC-based pericentromeric panels. Each panel consists of a core, which with a high resolution covers the most proximal euchromatic $\sim 0.7 \mathrm{Mb}$ (on average) of each chromosome arm and generally bridges the heterochromatin/euchromatin junction, as well as clones located proximally and distally to the core. The pericentromeric clone set was subsequently validated by the characterization of 19 sSMCs. Using the core probes, we could rapidly distinguish between heterochromatic (1/19) and euchromatic (11/19) SSMCs, and estimate the euchromatic DNA content, which ranged from approximately 0.13 to more than $10 \mathrm{Mb}$. The characterization was not completed for seven sSMCs due to a lack of information about the covered region in the reference sequence (1/19) or sample insufficiency (6/19).

(Continued on next page)
\end{abstract}

\footnotetext{
* Correspondence: c.castronovo@auxologico.it

${ }^{1}$ Laboratorio di Citogenetica Medica e Genetica Molecolare, IRCCS Istituto

Auxologico Italiano, via Ariosto 13, 20145, Milano, Italy

Full list of author information is available at the end of the article
} 
(Continued from previous page)

Conclusions: Our results demonstrate that this pericentromeric clone set is useful as an alternative tool for sSMC characterization, primarily in cases of very small SMCs that contain either heterochromatin exclusively or a tiny amount of euchromatic sequence, and also in cases of low-level or cryptic mosaicism. The resulting data will foster knowledge of human proximal euchromatic regions involved in chromosomal imbalances, thereby improving genotype-phenotype correlations.

Keywords: Small supernumerary marker chromosomes, Pericentromeric clone set, Heterochromatin/euchromatin boundary, FISH analysis, Array CGH analysis, Phenotype prediction

\section{Background}

Small supernumerary marker chromosomes (sSMCs) are additional centric chromosomal segments that are difficult to characterize by conventional cytogenetics alone due to their small size [1]. Excluding the sSMCs associated with five well-known syndromes (Pallister-Killian, isodicentric chromosome 15q [2], isochromosome 18p, Cat Eye $[\mathrm{i}(22 \mathrm{p} \sim \mathrm{q})]$, and derivative chromosome 22 $[\operatorname{der}(22) t(11 ; 22)]$ syndromes [1]), the overall risk for a pathological phenotype in prenatal de novo cases is 26-30\% [3].

The phenotypic expression of sSMCs ranges from asymptomatic to symptomatic, and depends on several factors including chromosomal origin, satellite vs. nonsatellite inclusion, euchromatic/heterochromatic content, uniparental disomy (UPD) of the chromosomes homologous to the sSMC, and mosaicism [3]. Furthermore, the presence of centromere-proximal euchromatin on an SSMC correlates with abnormal phenotypes, although several exceptions have been described [4]. Since the optimal strategies for genetic counseling and clinical management depend on the characteristics of sSMCs, it is vitally important to precisely characterize sSMCs in order to obtain additional information regarding their phenotypic effects. To this end, several fluorescent in situ hybridization (FISH)-based techniques have been developed over the years [5] for determining the origin of sSMCs and allowing breakpoint characterization, at least in cases of larger euchromatic SMCs. These methods include multicolor FISH (M-FISH) [6], spectral karyotyping (SKY) [7], centromere- and subcentromere-specific M-FISH (cenM-FISH and subcenM-FISH) [3,8,9], multicolor banding [10], and microdissection followed by reverse FISH [11,12]. More recently, a pericentric-ladderFISH (PCL-FISH) probe set has been developed based on 174 locus-specific BAC probes, and this probe set has been used in dual-color/multicolor-FISH approaches. This tool is specific for the pericentromeric regions and, therefore, enables sSMC breakpoint characterization with a resolution between 1 and $\sim 10 \mathrm{Mb}$ [13].

Furthermore, array-based comparative genomic hybridization (array CGH) analysis has been extensively used in sSMC characterization. This method allows, in a single experiment, determination of the marker chromosomal origin, definition of the size of aberrations (including euchromatic regions), and identification of complex rearrangements or multiple markers in single individuals [14-20]. However, array CGH may fail to identify the origins of very small SMCs in up to $50 \%$ of cases because its pericentromeric coverage is limited to the presence of segmental duplications, and it may also be unable to detect low-level and cryptic mosaicism [13,19-21]. Consequently, it is necessary to complement array CGH using FISH approaches [13,22]. In addition, to allow rapid discrimination between sSMCs that are positive or negative for unique sequences, an alternative approach using multiplex ligation-dependent probe amplification (MLPA) analysis has recently been developed for use in the context of prenatal diagnosis [23].

In this study, we report the design and validation of a new pericentromeric Bacterial Artificial Chromosome (BAC) clone set that covers the most proximal euchromatic sequences of all human chromosome arms, as well as the heterochromatin/euchromatin junctions, excluding the short arms of acrocentric chromosomes. This set was designed to improve molecular characterization of sSMCs by FISH analysis, a molecular-cytogenetic technique that, in contrast to array $\mathrm{CGH}$, is available in most cytogenetic laboratories. This new complementary tool will be especially useful in cases of low-level mosaicism and/or very small marker chromosomes, which are likely to consist entirely of heterochromatin or contain only a tiny amount of euchromatic sequence, as demonstrated by some reported sSMC cases.

\section{Results}

Setting up 43 pericentromeric BAC probe panels

Using FISH analysis, we assayed 561 pericentromeric BAC probes, of which 323 (57.6\%) were specific, 163 (29.0\%) exhibited multiple cross-hybridizations, and 75 (13.4\%) were excluded because of a wrong chromosomal localization. We then established 43 pericentromeric panels (excluding the acrocentric $\mathrm{p}$ arms), each of which consists of a high resolution core panel, which bridges the heterochromatin/euchromatin junction and usually comprises 3-7 contiguous or very close clones, as well as 
clones located proximally and distally to the core (Additional file 1: Table S1). The core panels were completed for 35 chromosome arms $(2 \mathrm{p}, 2 \mathrm{q}, 3 \mathrm{p}, 3 \mathrm{q}$, $4 p, 4 q, 5 p, 5 q, 6 p, 6 q, 7 p, 7 q, 8 p, 8 q, 9 q, 10 p, 11 q, 12 p$, $12 \mathrm{q}, 13 \mathrm{q}, 14 \mathrm{q}, 15 \mathrm{q}, 16 \mathrm{p}, 16 \mathrm{q}, 17 \mathrm{p}, 17 \mathrm{q}, 18 \mathrm{p}, 18 \mathrm{q}, 19 \mathrm{p}$, 19q, 20p, 22q, Xp, Xq, and Yq); they span, generally in a continuum, the proximal $\sim 0.73 \mathrm{Mb}$ (on average) of each chromosome arm (Table 1). A core panel for chromosome Yp was not established because of the lack of a physical map in the reference sequence, whereas the core panels of seven other chromosome arms were incomplete due to either the lack of a heterochromatin/euchromatin junction in the reference sequence $(1 p, 10 q, 11 p, 20 q$, and $21 q)$, or the presence of pericentromeric paralogous segmental duplications ( $1 \mathrm{q}$ and $9 \mathrm{p}$ ) that prevented us from obtaining unique mapping information (Table 1 and Additional file 1 : Table S1).

\section{Molecular-cytogenetic characterization of 19 sSMCs}

The utility of the clone set was then validated by the molecular characterization of 19 sSMCs, six of which were ascertained during routine prenatal testing (Additional file 2: Table S2). Nine sSMCs ( 47\%) were derived from acrocentric chromosomes, six ( 32\%) were inherited (patients 6, 8, 10-13), of which two were detected in two siblings (patients 10 and 11) and are complex marker chromosomes originating from a maternal balanced rearrangement. Multiple markers occurred in a single adult patient (5\%) (patient 15). Mosaicism was detected in seven patients ( $37 \%)$ (patients $1-5,15$, and 18), in most cases involving non-acrocentric chromosomes. Uniparental disomy (UPD) analysis of sSMC sister chromosomes was not performed. Details of sSMC characterization are provided in Additional file 2: Table S2.

Using the core panel probes, we were able to rapidly distinguish between heterochromatic (1/19) and pericentromeric euchromatic marker chromosomes (11/19), even in cases of low-level mosaicism. In addition, we either precisely established or estimated the size of the euchromatic content in 17 out of 19 sSMCs (Additional file 2: Table S2, Figures 1, 2 and 3). The euchromatic DNA present on the sSMCs ranged from $\sim 0.13 \mathrm{Mb}$ to more than $10 \mathrm{Mb}$ (Additional file 2: Table S2). However, sSMC characterization was not completed in patient 6 , due to the incompleteness of the chromosome 21q core panel physical map in the reference sequence, and in patients 7-11 and 15, due to sample insufficiency (Additional file 2: Table S2).

In two patients, we detected large marker chromosomes in high-level mosaicism, as follows: a ring chromosome 4 $[\mathrm{r}(4)]$ in patient 3, and an $\mathrm{r}(11)$ in patient 5 (Additional file 2: Table S2, Figure 2). The molecular characterization of these SMCs was performed using the panel probes and subsequently refined by array CGH analysis, which allowed a more precise definition of the breakpoints.
In particular, the chromosome 4p breakpoint was mapped between BAC probes RP11-89F4 and RP11-178N2 (chr4:44,621,297 - 45,736,237, hg19) (Figure 2B), and then narrowed down by array CGH to a $236 \mathrm{~kb}$ region between oligonucleotide probes A_14_P127561 and A_14_P128650 (chr4:45,518,972 - 45,754,992, hg19); thus, the breakpoint was mapped at $45.5-45.7 \mathrm{Mb}$ from the 4p telomere (chr4:45,518,972-45,736,237, hg19) (Additional file 2: Table S2, Figure 2A). Similarly, the 4q breakpoint was mapped between BACs RP11-91C3 (Figure 2C) and RP11-63E13 (chr4:61,086,318-66,600,553, hg19), and the position was then refined to a location between oligonucleotides A_14_P137150 and A_14_P133582 (chr4:61,126,608-61,343,360, hg19) (Additional file 2: Table S2, Figure 2A). For sSRC(11), the 11p breakpoint was mapped between BACs RP11-1062A8 and RP11746P9 (chr11:49,259,387-50,111,641, hg19) (Figure 2E), and then refined between oligonucleotides A_14_P126799 and A_14_P134986 (chr11:49,919,441-50,378,743, hg19); thus, this breakpoint mapped precisely at $49.9-50.1 \mathrm{Mb}$ from the 11p telomere (chr11:49,919,441-50,111,641, hg19) (Additional file 2: Table S2, Figure 2D). The 11q breakpoint was characterized only by array CGH because the 11q panel probes were not informative due to their proximal position (Additional file 2: Table S2, Figure 2D,F). No further cryptic genomic imbalances were detected in either patient.

\section{Discussion}

Pericentromeric regions of human chromosomes are transitional territories between centromeric heterochromatin and euchromatic regions. They represent complex mosaic structures, including coding sequences interspersed with non-coding sequences [24]. Therefore, sequencing of these regions is technically difficult, and a complementary approach is necessary to clarify their role in human disease.

sSMCs generally contain a centromeric/pericentromeric region, and their precise characterization is a powerful tool for identifying which genomic regions lead to abnormalities when they are affected by dosage imbalances. Over the years, numerous FISH-based approaches have been developed, and these methods have contributed to improvements in SSMC characterization. However, assays such as M-FISH/SKY [6,7], cenM-FISH [8] and subcenMFISH $[3,9]$ are limited to the identification of the sSMC chromosomal origin or the characterization of larger euchromatic SMCs, and only the use of FISH-banding or locus-specific probes $[13,25,26]$ can improve breakpoint characterization $[13,27,28]$. In particular, the PCL-FISH probe set, recently developed by Hamid et al., (2012) [13], is a bar-code FISH assay that constitutes a $10 \mathrm{Mb}$ raster along pericentromeric chromosomal regions, allowing the determination of mosaic and non-mosaic sSMC 
Table 1 Pericentromeric euchromatic coverage of the high-resolution BAC-based core panels

\begin{tabular}{|c|c|c|c|c|c|c|}
\hline Chromosome arm & $\begin{array}{l}\text { Heterochromatic } \\
\text { region starting } \\
\text { position }(\mathrm{Mb})^{a}\end{array}$ & $\begin{array}{l}\text { Heterochromatic } \\
\text { region ending } \\
\text { position }(\mathrm{Mb})^{a}\end{array}$ & $\begin{array}{l}\text { Proximal euchromatic } \\
\text { BAC clone belonging } \\
\text { to the core panel }\end{array}$ & $\begin{array}{l}\text { Distance between heterochromatic } \\
\text { region and proximal euchromatic } \\
\text { core BAC clone }(\mathrm{Mb})^{b}\end{array}$ & $\begin{array}{l}\text { Distal euchromatic } \\
\text { BAC clone belonging } \\
\text { to the core panel }\end{array}$ & $\begin{array}{l}\text { Distance between proximal } \\
\text { and distal euchromatic core } \\
\text { BAC clones (Mb) }\end{array}$ \\
\hline $1 p^{d}$ & 121.5 & & RP11-803J8 at $1 p 11.2$ & 0.015 & CTD-3138A9 at 1p12-p11.2 & 0.98 \\
\hline $1 q^{d}$ & & 142.6 & RP11-15M9 at 1q21.1 & 0.76 & CTD-2326L14 at 1q21.1 & 1.46 \\
\hline $2 p$ & 90.5 & & CTD-2269O20 at 2p11.2-p11.1 & 0 & $\mathrm{RP} 11-1023 \mathrm{H} 22$ at 2p11.2 & 0.61 \\
\hline $2 q$ & & 96.8 & RP11-139J5 at 2q11.1-q11.2 & 0 & RP11-245P4 at 2q11.2 & 0.96 \\
\hline $3 p$ & 87.9 & & RP11-424C9 at 3p11.2-p11.1 & 0 & RP11-598A10 at 3p12.1-p11.2 & 0.71 \\
\hline $3 q$ & & 93.9 & RP11-259L20 at 3q11.1-q11.2 & 0 & RP11-625F19 at $3 q 11.2$ & 0.87 \\
\hline $4 p$ & 48.2 & & RP11-260K18 at 4p12-p11 & 0 & CTD-2057N12 at 4p12 & 0.88 \\
\hline $4 q$ & & 52.7 & RP11-98B6 at 4q11-q12 & 0 & RP11-600M5 at 4q12 & 0.76 \\
\hline $5 p$ & 46.1 & & RP11-10F16 at 5p12-p11 & 0 & RP11-134N5 at 5p12 & 0.91 \\
\hline $5 q$ & & 50.7 & RP11-463E10 at 5q11.1-q11.2 & 0 & CTD-3113M11 at $5 q 11.2$ & 0.33 \\
\hline $6 p$ & 58.7 & & RP11-136G2 at 6p11.2-p11.1 & 0 & RP11-799H20 at $6 \mathrm{p} 11.2$ & 1.02 \\
\hline $6 q$ & & 63.3 & RP11-448N11 at 6q11.1-q11.2 & 0 & RP11-78B14 at $6 q 12$ & 0.30 \\
\hline $7 p$ & 58.0 & & CTD-2593N8 at 7p11.2-p11.1 & 0 & RP11-114G11 at 7p11.2 & 0.66 \\
\hline $7 q$ & & 61.7 & CTD-224501 at 7q11.1-q11.21 & 0 & RP11-45N18 at 7q11.21 & 0.84 \\
\hline $8 p$ & 43.1 & & RP11-73M19 at 8p11.21-p11.1 & 0 & RP11-577C12 at 8p11.21 & 0.79 \\
\hline $8 q$ & & 48.1 & CTD-2563N10 at 8q11.1-q11.21 & 0 & RP11-367A12 at 8q11.21 & 0.56 \\
\hline $9 p^{d}$ & 47.3 & & RP11-606B18 at 9p11.2 & 0.37 & RP11-361G22 at 9p11.2 & 0.64 \\
\hline $9 q$ & & 65.9 & CTD-2508M5 at 9q12-q13 & 0 & CTD-2050L17 at 9q13 & 0.49 \\
\hline 10p & 38.0 & & CTD-3195G22 at 10p11.21-p11.1 & 0 & RP11-739D18 at 10p11.21 & 0.58 \\
\hline $10 q^{d}$ & & 42.3 & RP11-80L2 at $10 \mathrm{q} 11.21$ & 0.08 & RP11-351D16 at 10q11.21 & 1.32 \\
\hline $11 p^{d}$ & 51.6 & & RP11-100E23 at $11 \mathrm{p} 11.12$ & 0.06 & RP11-318O24 at $11 \mathrm{p} 11.12$ & 1.00 \\
\hline $11 q$ & & 55.7 & CTD-3202L3 at 11q11-q12.1 & 0 & RP11-720L5 at 11q12.1 & 0.85 \\
\hline $12 p$ & 33.3 & & RP11-460N10 at 12p11.21-p11.1 & 0 & RP11-8P13 at 12p11.21 & 0.48 \\
\hline $12 q$ & & 38.2 & RP11-496H24 at 12q11-q12 & 0 & RP11-715M8 at 12q12 & 0.68 \\
\hline $13 q$ & & 19.5 & RP11-294G16 at 13q11-q12.11 & 0 & RP11-7111 at $13 q 12.11$ & 0.32 \\
\hline $14 q$ & & 19.1 & RP11-639F13 at 14q11.1-q11.2 & 0 & RP11-77E23 at 14q11.2 & 0.90 \\
\hline $15 q$ & & 20.7 & RP11-108C1 at 15q11.1-q11.2 & 0 & RP11-666L22 at $15 q 11.2$ & 0.34 \\
\hline $16 p$ & 34.6 & & RP11-488I20 at 16p11.2-p11.2 & 0 & RP11-1088B6 at 16p11.2 & 0.32 \\
\hline $16 q$ & & 47.0 & RP11-627O2 at 16q11.2-q12.1 & 0 & RP11-671L23 at 16q12.1 & 0.36 \\
\hline $17 p$ & 22.2 & & RP11-718K3 at 17p11.2-p11.1 & 0 & RP11-937K3 at 17p11.2 & 0.51 \\
\hline $17 q$ & & 25.8 & RP11-1049N9 at $17 \mathrm{q} 11.1-\mathrm{q} 11.2$ & 0 & RP11-59G20 at 17q 11.2 & 0.52 \\
\hline
\end{tabular}


Table 1 Pericentromeric euchromatic coverage of the high-resolution BAC-based core panels (Continued)

\begin{tabular}{|c|c|c|c|c|c|c|}
\hline $18 p$ & 15.4 & & RP11-1133K23 at 18p11.21-p11.1 & 0 & RP11-1025M21 at 18p11.21 & 0.52 \\
\hline $18 q$ & & 19.0 & RP11-746M23 at $18 q 11.1-q 11.2$ & 0 & $\mathrm{RP} 11-60 \mathrm{G} 3$ at $18 \mathrm{q} 11.2$ & 0.66 \\
\hline $19 p$ & 24.4 & & RP11-350E11 at 19p12-p11 & 0 & RP11-642/13 at 19p12 & 0.86 \\
\hline $19 q$ & & 28.6 & CTD-2045N7 at $19 q 11-q 12$ & 0 & RP11-34716 at $19 q 12$ & 0.95 \\
\hline $20 p$ & 25.6 & & RP11-161K13 at 20p11.21-p11.1 & 0 & RP11-156D15 at 20p11.21 & 0.49 \\
\hline $20 q^{d}$ & & 29.4 & CTD-2311M18 at 20q11.21 & 0.02 & RP11-1147|19 at 20q11.21 & 1.17 \\
\hline $21 q^{d}$ & & 14.3 & RP11-203F20 at 21q11.2 & 0.13 & RP11-1025M7 at 21q11.2 & 0.93 \\
\hline $22 q$ & & 17.9 & RP11-958H20 at 22q11.1-q11.21 & 0 & RP11-81B3 at 22q11.21 & 0.71 \\
\hline$X p$ & 58.1 & & CTD-2225J11 at Xp11.21-p11.1 & 0 & RP11-936C8 at Xp11.21 & 0.96 \\
\hline $\mathrm{Xq}$ & & 63.0 & RP11-943J20 at Xq11.1-q11.2 & 0 & $\mathrm{RP} 11-284 \mathrm{~B} 18$ at $\mathrm{Xq11.2}$ & 0.54 \\
\hline$Y p^{e}$ & 11.6 & & RP11-108114 at Yp11.2 & 1.50 & & \\
\hline$Y q$ & & 13.4 & RP11-1100G7 at Yq11.1-q11.21 & 0 & RP11-91N9 at Yq11.21 & 1.1 \\
\hline
\end{tabular}

${ }^{a}$ The location of the heterochromatic region is an approximation taken from the February 2009 release of the UCSC Human Genome Browser Database, which indicates the distance from the most distal available $\mathrm{p}-$ arm sequence to the centromere start and end for that chromosome. For chromosomes 1q, 9q, and 16q, the heterochromatic region ending position indicates the telomeric border of the heterochromatic bands 1q12, $9 \mathrm{q} 12$, and $16 \mathrm{q} 11.2$, respectively.

${ }^{b}$ The distance between the heterochromatic region and the most proximal available euchromatic BAC clone indicates the starting position of the pericentromeric coverage of the BAC core panel for each chromosome arm.

'The distance between the proximal and distal euchromatic core BAC clones indicates the pericentromeric euchromatic coverage of the core panel for each chromosome arm; if the proximal BAC clone overlaps with heterochromatin/euchromatin bridge, the distance refers only to the euchromatic sequence.

${ }^{d}$ The core panels of chromosomes $1 \mathrm{p}, 10 \mathrm{q}, 11 \mathrm{p}, 20 \mathrm{q}$, and $21 \mathrm{q}$ were not completed because of the lack of heterochromatin/euchromatin-bridging physical maps in the reference sequence. The core panels of

chromosomes $1 q$ and $9 p$ are incomplete due to the presence of pericentromeric paralogous segmental duplications; therefore, the corresponding core panel probes were excluded from the clone set because their hybridization signals do not give unique mapping information. The partial core panel of chromosome 10q was established using non-contiguous clones.

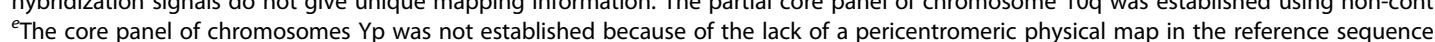




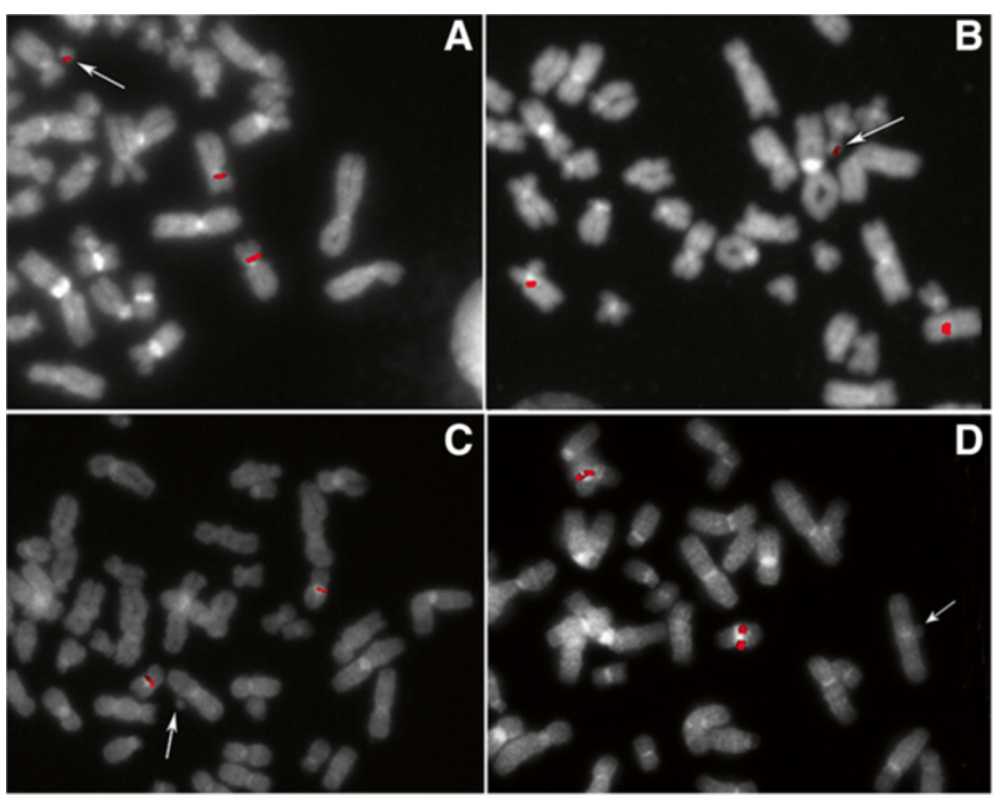

Figure 1 FISH characterization of $\operatorname{sSRC}(10)$ of patient $4(A, B)$ and $\operatorname{SSRC}(16)$ of patient $14(C, D)$, including breakpoint mapping and sizing of the euchromatic region. (A) Partial metaphase hybridized with the 10p11.23 BAC probe RP11-39E10, showing signals on both chromosome 10 homologs and the der(10) marker chromosome (arrow). (B) Partial metaphase hybridized with the BAC probe RP11-178A10 at 10q11.21, showing a diminished hybridization signal on the der(10) marker chromosome (arrow) relative to the chromosome 10 homologs. (C and D) Partial metaphases hybridized with the BAC probes RP11-488120 at 16p (C) and CTD-2382P11 at 16q (D), showing signals on the chromosome 16 homologs but not SSRC(16) (arrows).

breakpoints within genomic regions of $1-10 \mathrm{Mb}$ in size. In addition, this approach has been particularly useful in characterizing cryptic mosaic sSMCs [29], and for easily defining all involved breakpoints [13]. However, because it covers the most proximal $1 \mathrm{Mb}$ of euchromatic sequences of each chromosome arm with a very low resolution, PCL-FISH is not useful for the characterization of very small SMCs that contain only tiny amounts of euchromatic sequence [13]. Likewise, a more sensitive technique such as array $\mathrm{CGH}$, which can significantly narrow down sSMC breakpoints [14-20,30], can still yield incomplete pericentromeric coverage due to the presence of large duplicated sequences. Moreover, array CGH cannot detect low-level and/or cryptic mosaic sSMCs.

Therefore, in order to provide a complementary tool for SSMC characterization, we established a FISH-based pericentromeric BAC clone set, including probes that cover the most proximal euchromatic $\sim 0.7 \mathrm{Mb}$ (on average) of each chromosome arm at a high resolution. The pericentromeric probe set also includes probes that bridge the heterochromatin/euchromatin junctions (Table 1 and Additional file 1: Table S1), enabling rapid discrimination between euchromatic (11/19 in the present series) and heterochromatic (1/19) sSMCs, irrespective of marker chromosome origin (Additional file 2: Table S2). The most proximal probes were chosen independently of the presence of segmental duplications, with the exceptions of chromosomes $1 \mathrm{q}$ and $9 \mathrm{p}$, where pericentromeric paralogous segmental duplications have been detected. As expected, a significant percentage of the assayed probes $(\sim 13 \%)$ were mislocalized, supporting the need for the large screening effort we performed to verify the predicted physical position of each clone. As previously reported for PCL-FISH [13], we confirmed the utility of our new BAC-probe set in characterizing low-level mosaic sSMCs (Additional file 2: Table S2), suggesting that this approach could be applied to breakpoint identification in cases of cryptic mosaic sSMCs; however, no pertinent cases are present in the series reported here.

In terms of genotype-phenotype correlation, the data we collected regarding sSMC characterization allowed us to confirm the existence of pericentromeric euchromatic critical and noncritical regions surrounding the centromeres of all chromosome arms, i.e., regions in which trisomy or tetrasomy either does or does not correlate with pathological phenotypes [4]. Accordingly, we declare no clinical signs reported in association with acrocentric sSMCs that have breakpoints localized in predicted pericentromeric noncritical regions $[4,14,16,22,31]$ (patients 6-9, 12, and 13), with the exception of patient 7, who exhibited a growth delay likely not associated with the sSMC (Additional file 2: Table S2). Furthermore, in siblings 10 and 11 , the $14 \mathrm{q}$ breakpoint characterization was useful in linking the reported clinical findings specifically to trisomy of 6pter-p25 (Additional file 2: Table S2). By contrast, we classified the larger idic(22;22) marker 

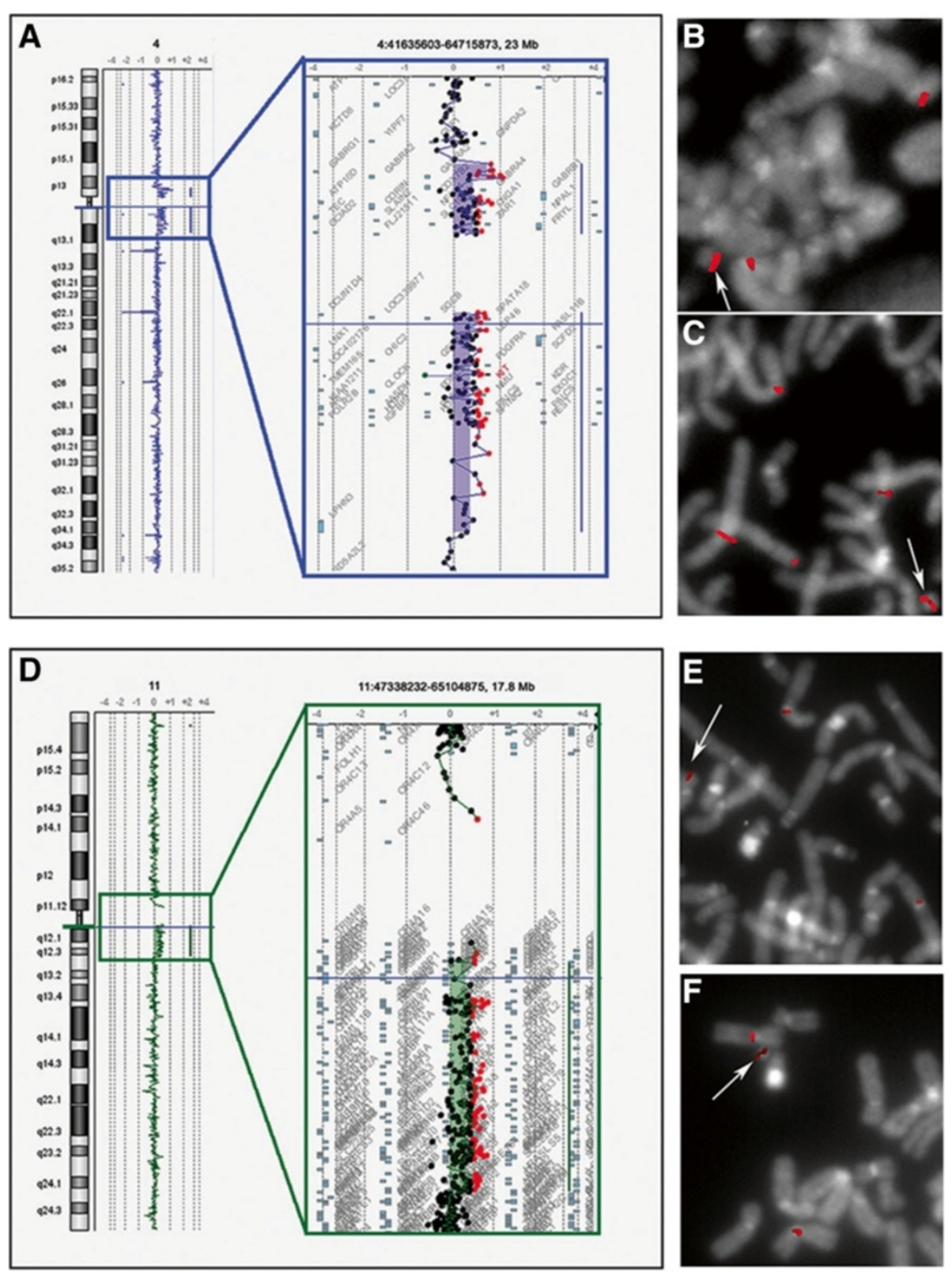

Figure 2 Combined array-CGH and FISH characterization of SRC(4) of patient 3 (A-C) and SRC(11) of patient 5 (D-F). Upper row: (A) Identification of a heterozygous duplication of $15.4 \mathrm{Mb}$ (chr4:45,754,992-61,126,608, hg19) using array CGH (Agilent $4 \times 44 \mathrm{~K})$ in patient 3 . The BAC probes RP11-178N2 at 4p12 (B) and RP11-91C3 at 4q13.1 (C) produce hybridization signals on both chromosomes 4 and the SRC(4). Bottom row: (D) Identification of a heterozygous duplication of 13.4 Mb (chr11:50,378,743-63,743,029, hg19) using array CGH (Agilent $4 \times 44 \mathrm{~K}$ ) in patient 5. The BAC probes RP11-746P9 at 11p11.12 (E) and RP11-872D17 at $11 \mathrm{q} 12$ (F) produce hybridization signals on both chromosomes 11 and the SRC(11).

chromosomes (patients 16-18) as type I Cat Eye Syndrome (CES) chromosomes, which results in the CES phenotype [32], as confirmed in patient 17 (Additional file 2: Table S2). In addition, in patient 18, the molecular characterization of idic(22;22) revealed asymmetrical breakpoints that resulted in both a tetrasomy of $\sim 750 \mathrm{~kb}$, which featured the gap reported between the end of the noncritical region and the start of the critical region [4], and a trisomy of $\sim 150-400 \mathrm{~kb}$ of euchromatic sequences that are included within the $22 \mathrm{q}$ predicted critical region [4] (Additional file 2: Table S2, Figure 3E,F). These observations suggest that the mild phenotype of patient 18 , relative to the classical CES clinical presentation, can be at least partially attributed to trisomy rather than tetrasomy of the same euchromatic region, combined with low-level mosaicism of sSMC(22) (Additional file 2: Table S2).

Among the non-acrocentric marker chromosomes collected in our series, the SMC characterization revealed 

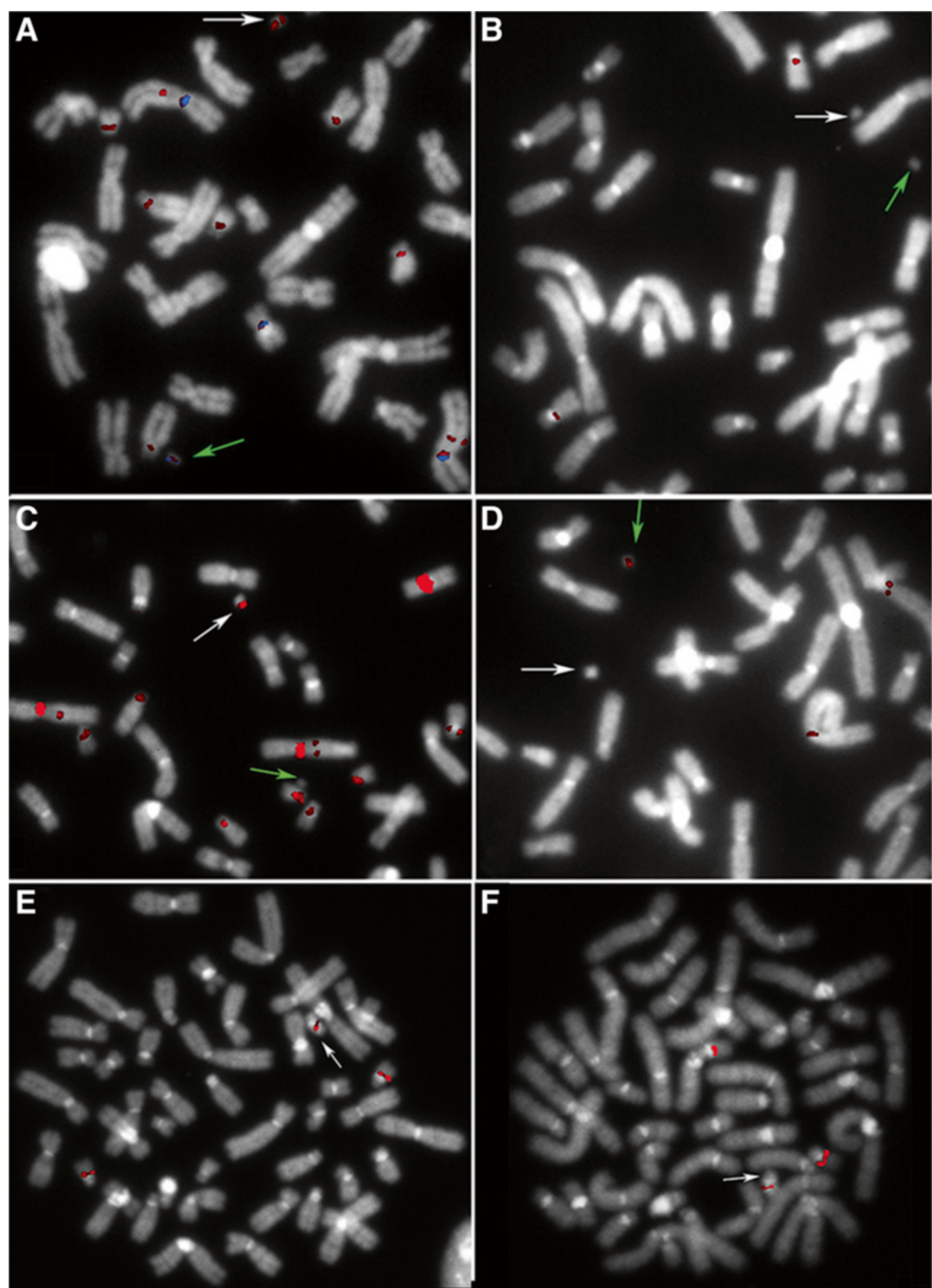

Figure 3 FISH characterization of $\operatorname{sSMC}(2)$ and $\operatorname{SSMC}(18)$ of patient 15 (A-D), and $\mathrm{sSMC}(22)$ of patient 18 (E, F). (A) Partial metaphase hybridized with the BAC probe RP11-749K13 at 18p11.21 (red), showing a hybridization signal on idic(18;18) marker chromosomes (white arrow) that is enlarged relative to those on chromosome 18 homologs, as well as cross-hybridization signals on the der(2) marker chromosome (green arrow) and chromosome 2 homologs. The BAC probe RP11-1069D4 at 2p11.1 (blue) produces hybridization signals on der(2) marker chromosome (green arrow) and chromosome 2 homologs. (B) Partial metaphase hybridized with the BAC probe RP11-1035O2 at 18q11.1 does not show a hybridization signal on $\operatorname{idic}(18 ; 18)$ (white arrow). (C) Partial metaphase hybridized with the BAC probe RP11-134N21 at 2q11.1, which is deleted on der(2) (green arrow), showing cross-hybridization signals on both idic(18;18) (white arrow) and chromosome 18 homologs. (D) Partial metaphase hybridized with the BAC probe RP11-71B7 at 2q11.1, showing a hybridization signal on der(2) (green arrow) and on chromosome 2 homologs.

(E) Metaphase hybridized with the BAC probes RP11-1053O2 at 22q11.21 shows an equal signal on the idic(22;22) marker chromosome (arrow) relative to the signals on the chromosome 22 homologs. (F) Metaphase hybridized with the BAC probe RP11-690P21 at 22q11.21 shows a diminished signal on the idic(22;22) marker chromosome (arrow) relative to the signals on the chromosome 22 homologs.

breakpoints within pericentromeric noncritical regions in two cases [4,14-16,20,22,33,34], consistent with the observation that the corresponding patients (3 and 15) had normal phenotypes (Figures $2 \mathrm{~A}-\mathrm{C}$ and $3 \mathrm{~A}-\mathrm{D}$, Additional file 2: Table S2). Notably, the association of sSMC(2) and sSMC(18) observed in patient 15 has not 
been previously reported. By performing FISH analysis, we identified pericentromeric segmental duplications shared by chromosomes 2 and 18 (Figure 3A-D), and hypothesized that a complex genomic rearrangement had occurred between those two chromosomes, resulting in SSMC formation and in the simultaneous deletion of the sequence covered by probe RP11-134N21 from sSMC(2) (Figure 3D, Additional file 2: Table S2).

In regard to pathological non-acrocentric sSMCs, our molecular characterization helped us to either refine or confirm the boundaries of the predicted critical regions and to improve genotype-phenotype correlations. For example, characterization of patient 2's mosaic $\operatorname{sSRC}(1)$ indicated possible trisomy of 1p12-q21.1 chromosomal region, involving at most $\sim 1.91 \mathrm{Mb}$ of euchromatic sequences at 1q21.1 (Additional file 2: Table S2). Although the boundaries between the critical and noncritical regions on 1q are not yet available [4], we propose that trisomy of 1q21.1 might be responsible for patient 2's phenotype (Additional file 2: Table S2), as suggested by previous data [3,9,35-38]. However, neurological abnormalities like those exhibited by our patient have been previously reported in patients carrying mosaic sSMC (1), resulting in trisomy of the $1 \mathrm{p} 12-\mathrm{q} 12$ region. In those cases, the 1q breakpoints were mapped within the 1q12 heterochromatic region, suggesting that the $1 \mathrm{p}$ trisomy was responsible for the pathological phenotype $[3,8,9,39]$.

Both breakpoints of patient 5's SRC(11) were inferred to have occurred in the pericentromeric critical regions, leading to mosaic trisomy of the $11 \mathrm{p} 11.12-\mathrm{q} 13.1$ region (Additional file 2: Table S2, Figure 2E,F). To date, only one patient (11-W-p11.12/3-1 in the sSMC database [4]) has been reported to have a mosaic SSRC(11) characterized by breakpoints mapped within the same chromosomal bands found in our patient [4]. The reported pathological clinical presentation, characterized by dysmorphism and severe developmental delay, resembles that observed in patient 5 (Additional file 2: Table S2), confirming the pathogenetic role of mosaic trisomy of 11p11.12-q13.1.

Finally, sSMC(10) of patient 4 and $\operatorname{sSRC}(16)$ of patient 14 were both ascertained during prenatal testing. The sSRC(16) exclusively involved centromeric heterochromatin (Additional file 2: Table S2, Figure 1C, D), leading the parents to continue the pregnancy, which ended with the birth of a normal baby. By contrast, the sSMC (10) resulted in mosaic trisomy of the 10p11.23-q11.21 region (Additional file 2: Table S2, Figure 1A,B). We considered that a pathological phenotype might arise due to trisomy of at least $3.6 \mathrm{Mb}$ of $10 \mathrm{p}$ proximal euchromatic sequences because the $10 \mathrm{q}$ breakpoint mapped in the predicted noncritical region $[4,17,40]$. This hypothesis was subsequently confirmed by the patient's neonatal phenotype (Additional file 2: Table S2).

\section{Conclusions}

To summarize, our data demonstrate the potential value of our pericentromeric clone set for characterization of sSMCs in both prenatal and postnatal diagnostics. Due to the fact that the established resource covers all available pericentromeric regions, it may be particularly useful in cases of very small marker chromosomes, allowing rapid discrimination between heterochromatic and euchromatic sSMCs, as well as precise sizing of imbalances. We also demonstrated the complementarity of FISH analysis using the pericentromeric clone set with array $\mathrm{CGH}$ analysis in the characterization of large marker chromosomes.

Apart from ad hoc combinations of different methods, performed when requested, FISH analysis is currently the only available technique for analyzing sSMCs in low or cryptic mosaicism. However, to use this probe set in prenatal cases, cytogenetic labs need to have sufficient resources to store the BAC clones and prepare the probes within a short time. Therefore, although application of this tool in prenatal diagnosis would be beneficial, we strongly recommend that it is used in research aimed at increasing our knowledge of the imbalances of human proximal euchromatic regions, thereby improving genotype-phenotype correlations and the assessment of the genetic risks of supernumerary marker chromosomes.

\section{Methods}

\section{sSMC samples}

Chromosomal samples from peripheral blood lymphocytes and amniotic fluid were collected from five cytogenetic labs. All samples were previously karyotyped by conventional cytogenetics (Q- or G-banding), and the sSMC origin was previously identified by FISH analysis using commercial centromere-specific probes (Vysis, Maidenhead, UK), following the manufacturer's instructions. In both cases, at least 16 metaphases were analyzed.

\section{FISH analysis}

RPCI-11 and CTD BAC clones covering the pericentromeric genomic regions of human chromosome arms were selected by consulting the UCSC Genome Browser Database (http://genome.cse.ucsc.edu, hg19, February 2009). Clones were provided by Prof. Mariano Rocchi, University of Bari (IT) and Invitrogen (Carlsbad, CA, USA). The probes' physical positions were verified on a few control metaphases derived from peripheral blood lymphocytes. All probes were labeled by nick-translation with biotin (Hoffman-La Roche, Basel, Switzerland), digoxigenin (Hoffman-La Roche), or Cy3 (Amersham Biosciences, Chalfont St. Giles, UK), and then visualized using FITC-anti-digoxigenin antibodies (Hoffman-La Roche) or streptavidin-DEAC (Sigma-Aldrich, St. Louis, MO, USA). Image acquisition was performed on a Leica DMRA2 fluorescence microscope (Leica, Wetzlar, 
Germany) equipped with Leica filters specific for DAPI, FITC, Cy3, and DEAC. Images were acquired using a charge-coupled device (CCD) camera (Leica) with a magnification factor of $100 \times$. Image analysis was performed using the Leica CW4000-FISH software (version Y1.3.1). In the initial step of sSMC characterization, the most proximal available core probe(s) for the involved chromosome arm(s) was used to discriminate between euchromatic and heterochromatic sSMCs. Next, fine breakpoint mapping was performed using clones within and/or distal to the core panels, depending on SSMC size. A single-color, dual-color, or three-color hybridization approach was chosen depending on the available amount of sSMC sample and the time available to complete the analysis. In case of non-mosaic sSMCs, at least 16 metaphases were analyzed, whereas in cases of mosaic sSMCs, the number of analyzed metaphases decreased proportionally with the level of mosaicism. The FISH protocols of Lichter et al. [41] and Lichter and Cremer [42] were followed, with minor modifications.

\section{Array $\mathrm{CGH}$ analysis}

Array CGH analysis was performed using the Human Genome CGH Microarray Kit $4 \times 44 \mathrm{~K}$ (Agilent Technologies, Palo Alto, CA), which consists of 42,494 60-mer oligonucleotide probes covering the entire genome with an average spatial resolution of $\sim 43 \mathrm{~kb}$. From both test and sex-matched reference (Promega, Southampton, UK) samples, $3 \mu \mathrm{g}$ of genomic DNA, previously extracted from probands' whole blood using the GenElute ${ }^{\mathrm{TM}}$ Blood Genomic DNA kit (Sigma-Aldrich), was processed according to the manufacturer's protocol. Images were obtained using the Agilent Feature Extraction software (version 9.1), and chromosomal profiles were obtained using the ADM-2 algorithm provided by DNA Analytics software (v4.0) (Agilent Technologies).

\section{Consent}

Written informed consent to the research investigation, which was approved by the Ethical Clinical Research Committee of Istituto Auxologico Italiano, was obtained from either the adult patients or one of the parents in case of child patients.

\section{Additional files}

Additional file 1: Table S1. Detailed list of the collected 486 pericentromeric BAC probes, including the 214 clones which belong to the high-resolution core panels, according to the UCSC Genome Browser Database assembly hg19.

Additional file 2: Table S2. Summary of 19 sSMCs characterized using the pericentromeric BAC clone set, including clinical data of the carrying patients.

\section{Abbreviations}

Array $\mathrm{CGH}$ : Array comparative genomic hybridization;

CenM-FISH: Centromere-specific M-FISH; FISH: Fluorescence in situ hybridization; M-FISH: Multcolor-FISH; PCL-FISH: Pericentric-ladder-FISH; SKY: Spectral karyotyping; sSMC: Small supernumerary marker chromosomes; SubcenM-FISH: Subcentromere-specific M-FISH; UPD: Uniparental disomy.

\section{Competing interests}

The authors declare that they have no competing interests.

\section{Authors' contributions}

CC: study design, acquisition, analysis and interpretation of data from FISH and array-CGH analyses; manuscript preparation; VE: acquisition, analysis and interpretation of data from FISH analysis, review and approval of the manuscript final version; CM: acquisition, analysis and interpretation of data from array- $\mathrm{CGH}$, review and approval of the manuscript final version; TS: sample collection, karyotyping and SSMC origin characterization, review and approval of the manuscript final version; RL: sample and clinical data collection, karyotyping and SSMC origin characterization, review and approval of the manuscript final version; AMC: sample collection, karyotyping and SSMC origin characterization, review and approval of the manuscript final version; GS: sample collection, karyotyping and SSMC origin characterization, review and approval of the manuscript final version; RD: acquisition, analysis and interpretation of data from array-CGH; review and approval of the manuscript final version; BL: sample collection, karyotyping and SSMC origin characterization, review and approval of the manuscript final version; MD: clinical data collection, review and approval of the manuscript final version; GE: clinical data collection, review and approval of the manuscript final version; CP, clinical data collection, review and approval of the manuscript final version; GD, sample collection, karyotyping and sSMC origin characterization, review and approval of the manuscript final version; BMT, clinical data collection, review and approval of the manuscript final version; LL: study design, critical revision of the manuscript, approval of the final version; FP: study design, interpretation of experimental data, critical revision of the manuscript, approval of the final version. All authors read and approved the final manuscript.

\section{Authors' information}

CC: biologist, specialist in Medical Genetics, PhD degree in Experimental Pathology and Neuropathology, contract researcher at the Medical Cytogenetics and Molecular Genetics Lab, Istituto Auxologico Italiano, Milano, Italy; VE: biologist, specialist in Medical Genetics, currently fixed-term contract at the Cytogenetics Lab, Ospedale Niguarda Ca' Granda, Milano, Italy; CM: biotechnologist, student of the School of Specialty in Medical Genetics, contract researcher at the Medical Cytogenetics and Molecular Genetics Lab, Istituto Auxologico Italiano, Milano, Italy;

TS: biologist, specialist in Medical Genetics, permanent position at the Cytogenetics Lab, Istituti Ospitalieri, Cremona, Italy;

RL: biologist, specialist in Medical Genetics, manager at the Pre-and Post-Natal Diagnostic Unit of the Cytogenetics laboratory, Niguarda Ca' Granda Hospital, Milano, Italy;

AMC: biologist, specialist in Medical Genetics, permanent position at the Cytogenetics Lab, S. Giovanni Battista Hospital, Torino, Italy;

GS: biologist, specialist in Medical Genetics, head of the Cytogenetics Lab, Fondazione IRCCS Ca' Granda Ospedale Maggiore Policlinico, Milano, Italy; RD: biotechnologist, specialist in Medical Genetics, currently post-doc fellowship at the Medical Genetics lab, Department of Health Sciences, University of Milan;

BL: biologist, specialist in Medical Genetics, permanent position at the Medical Cytogenetics and Molecular Genetics Lab of Istituto Auxologico Italiano, Milano, Italy;

MD: medical doctor, specialist in Medical Genetics, permanent position at the Paediatric Unit, Fondazione IRCCS Ca' Granda Ospedale Maggiore Policlinico, Milano, Italy;

GE: medical doctor, specialist in Medical Genetics, permanent position at the Division of Medical Genetics, S. Giovanni Battista Hospital, Torino, Italy; CP: medical doctor, specialist in Medical Genetics, permanent position at the Division of Medical Genetics, Istituti Ospitalieri, Cremona, Italy; GD: biologist, specialist in Medical Genetics, head of the Cytogenetics Diagnostic Unit, Medical Cytogenetics and Molecular Genetics Lab, Istituto Auxologico Italiano, Milano, Italy; 
BMT: medical doctor, specialist in Medical Genetics, permanent position at the Division of Medical Genetics, San Luca Hospital, Istituto Auxologico Italiano, Milano, Italy;

LL: medical doctor, specialist in Medical Genetics, professor of Medical Genetics, Department of Health Sciences, University of Milano; research director of the Medical Cytogenetics and Molecular Genetics Lab, Istituto Auxologico Italiano, Milano, Italy;

FP: biologist, specialist in Medical Genetics, PhD degree in Genetics and Molecular Evolution, associate professor of Medical Genetics, Department of Medical Biotechnology and Translational Medicine, University of Milano; head of the Research Unit, Medical Cytogenetics and Molecular Genetics Lab, Istituto Auxologico Italiano, Milano, Italy.

\section{Acknowledgements}

The authors wish to thank Professor Mariano Rocchi and his team, supported by the Telethon Foundation, for having generously provided most of the clones used in this study. We also thank the patients and their families for their cooperation. The study was supported by a Ministry of Health grant to Istituto Auxologico Italiano IRCCS (Grant number: 08C506).

\section{Author details}

${ }^{1}$ Laboratorio di Citogenetica Medica e Genetica Molecolare, IRCCS Istituto Auxologico Italiano, via Ariosto 13, 20145, Milano, Italy. ${ }^{2}$ Servizio di Genetica, A.O. Istituti Ospitalieri, viale Concordia 1, 26100, Cremona, Italy. ${ }^{3}$ S.C. Anatomia, Istologia Patologica e Citogenetica, Ospedale Niguarda Ca' Granda, piazza dell'Ospedale Maggiore 3, 20162, Milano, Italy. ${ }^{4}$ S.C.D.U Genetica Medica, A.O.S. Giovanni Battista, corso Bramante 88, 10126, Torino, Italy. ${ }^{5}$ Laboratorio di Genetica Medica, Fondazione IRCCS Ca' Granda Ospedale Maggiore Policlinico, via Francesco Sforza 28, 20122, Milano, Italy. ${ }^{6}$ Clinica Pediatrica 1, Dipartmento di Patofisiologia e Trapianti, Università degli Studi di Milano, Fondazione IRCCS Ca' Granda Ospedale Maggiore Policlinico, via Francesco Sforza 28, 20122, Milano, Italy. ${ }^{7}$ Ambulatorio di Genetica Medica, Ospedale San Luca, IRCCS Istituto Auxologico Italiano, piazzale Brescia 20, 20149, Milano, Italy. ${ }^{8}$ Genetica Medica, Dipartimento di Scienze della Salute, Università degli Studi di Milano, via Antonio di Rudinì 8, 20142, Milano, Italy. ${ }^{9}$ Dipartmento di Biotecnologie Mediche e Medicina Traslazionale, Università degli Studi di Milano, via Viotti 3/5, 20133, Milano, Italy.

Received: 8 August 2013 Accepted: 8 October 2013 Published: 30 October 2013

\section{References}

1. Liehr T, Claussen U, Starke H: Small supernumerary marker chromosomes (sSMC) in humans. Cytogenet Genome Res 2004, 107:55-67.

2. Battaglia A: The inv dup (15) or idic (15) syndrome (tetrasomy $15 q$ ). Orphanet J Rare Dis 2008, 3:30.

3. Liehr T, Mrasek K, Weise A, Dufke A, Rodríguez L, Martínez Guardia N, Sanchís A, Vermeesch JR, Ramel C, Polityko A, Haas OA, Anderson J, Claussen U, von Eggeling F, Starke H: Small supernumerary marker chromosomes-progress towards a genotype-phenotype correlation. Cytogenet Genome Res 2006, 112:23-34.

4. Liehr T: Small supernumerary marker chromosomes (SSMC). Institute of Human Genetics and Anthropology, University of Jena. http://www.med. uni-jena.de/fish/sSMC/00START.htm. Accessed 08/08/2013.

5. Liehr T: Basics and literature on multicolor fluorescence in situ hybridization application. http://www.fish.uniklinikum-jena.de/mFISH.html.

6. Speicher MR, Gwyn Ballard S, Ward DC: Karyotyping human chromosomes by combinatorial multi-fluor FISH. Nat Genet 1996, 12:368-375.

7. Schröck E, du Manoir S, Veldman T, Schoell B, Wienberg J, Ferguson-Smith MA, Ning Y, Ledbetter DH, Bar-Am I, Soenksen D, Garini Y, Ried T: Multicolor spectral karyotyping of human chromosomes. Science 1996, 273:494-497.

8. Nietzel A, Rocchi M, Starke H, Heller A, Fiedler W, Wlodarska I, Loncarevic IF, Beensen V, Claussen U, Liehr T: A new multicolor-FISH approach for the characterization of marker chromosomes: centromere-specific multicolor-FISH (cenM-FISH). Hum Genet 2001, 108:199-204.

9. Starke H, Nietzel A, Weise A, Heller A, Mrasek K, Belitz B, Kelbova C, Volleth M, Albrecht B, Mitulla B, Trappe R, Bartels I, Adolph S, Dufke A, Singer S, Stumm M, Wegner RD, Seidel J, Schmidt A, Kuechler A, Schreyer I, Claussen U, von Eggeling F, Liehr T: Small supernumerary marker chromosomes (SMCs): genotype-phenotype correlation and classification. Hum Genet 2003, 114:51-67.

10. Liehr T, Starke H, Heller A, Kosyakova N, Mrasek K, Gross M, Karst C, Steinhaeuser U, Hunstig F, Fickelscher I, Kuechler A, Trifonov V, Romanenko SA, Weise A: Multicolor fluorescence in situ hybridization (FISH) applied to FISH-banding. Cytogenet Genome Res 2006, 114:240-244.

11. Engelen JJ, Albrechts JC, Hamers GJ, Geraedts JP: A simple and efficient method for microdissection and microFISH. J Med Genet 1998, 35:265-268.

12. Liehr T, Heller A, Starke H, Rubtsov N, Trifonov V, Mrasek K, Weise A, Kuechler A, Claussen U: Microdissection based high resolution multicolour banding for all 24 human chromosomes. Int J Mol Med 2002, 9:335-339.

13. Hamid AB, Kreskowski K, Weise A, Kosayakova N, Mrasek K, Voigt M, Santos Guilherme R, Wagner R, Hardekopf D, Pekova S, Karamysheva T, Liehr T, Klein E: How to narrow down chromosomal breakpoints in small and large derivative chromosomes - a new probe set. J Appl Genetics 2012, 53:259-269.

14. Baldwin EL, May LF, Justice AN, Martin CL, Ledbetter DH: Mechanisms and consequences of small supernumerary marker chromosomes: from barbara McClintock to modern genetic-counselling issues. Am J Hum Genet 2008, 82:398-410.

15. Ballif BC, Rorem EA, Sundin K, Lincicum M, Gaskin S, Coppinger J, Kashork CD, Shaffer LG, Bejjani BA: Detection of low-level mosaicism by array CGH in routine diagnostic specimens. Am J Med Genet A 2006, 140:2757-2767.

16. Ballif BC, Hornor SA, Sulpizio SG, Lloyd RM, Minier SL, Rorem EA, Theisen A, Bejjani BA, Shaffer LG: Development of a high-density pericentromeric region $\mathrm{BAC}$ clone set for the detection and characterization of small supernumerary marker chromosomes by array CGH. Genet Med 2007, 9:150-162.

17. Sheth F, Andrieux J, Ewers E, Kosyakova N, Weise A, Sheth H, Romana SP, LeLorc'h M, Delobel B, Theisen O, Liehr T, Nampoothiri S, Sheth J: Characterization of sSMC by FISH and molecular techniques. Eur J Med Genet 2011, 54:247-255.

18. Vetro A, Manolakos E, Petersen MB, Thomaidis L, Liehr T, Croci G, Franchi F, Marinelli M, Meneghelli E, Dal Bello B, Cesari S, lasci A, Arrigo G, Zuffardi O: Unexpected results in the constitution of small supernumerary marker chromosomes. Eur J Med Genet 2012, 55:185-190.

19. Reddy KS, Aradhya S, Meck J, Tiller G, Abboy S, Bass H: A systematic analysis of small supernumerary marker chromosomes using array $\mathrm{CGH}$ exposes unexpected complexity. Genet Med 2013, 15:3-13.

20. Marle N, Martinet D, Aboura A, Joly-Helas G, Andrieux J, Flori E, Puechberty J, Vialard F, Sanlaville D, Fert Ferrer S, Bourrouillou G, Tabet A, Quilichini B, Simon-Bouy B, Bazin A, Becker M, Stora H, Amblard S, Doco-Fenzy M, Molina Gomes D, Girard-Lemaire F, Cordier M, Satre V, Schneider A, Lemeur N, Chambon P, Jacquemont S, Fellmann F, Vigouroux-Castera A, Molignier R, et al: Molecular characterization of 39 de novo SSMC: contribution to prognosis and genetic counseling, a prospective study. Clin Genet 2013. 10.1111/cge.12138. [Epub ahead of print].

21. Bui $T H$, Vetro A, Zuffardi O, Shaffer LG: Current controversies in prenatal diagnosis 3: is conventional chromosome analysis necessary in the post-array CGH era? Prenat Diagn 2011, 31:235-243.

22. Manolakos E, Kefalas K, Neroutsou R, Lagou M, Kosyakova N, Ewers E, Ziegler M, Weise A, Tsoplou P, Rapti SM, Papoulidis I, Anastasakis E, Garas A, Sotiriou S, Eleftheriades M, Peitsidis P, Malathrakis D, Thomaidis L, Kitsos G, Orru S, Liehr T, Petersen MB, Kitsiou-Tzeli S: Characterization of 23 small supernumerary marker chromosomes detected at pre-natal diagnosis: the value of fluorescence in situ hybridization. Mol Med Rep 2010, 3:1015-1022.

23. Van Opstal D, Boter M, Noomen P, Srebniak M, Hamers G, Galjaard RJ: Multiplex ligation dependent probe amplification (MLPA) for rapid distinction between unique sequence positive and negative marker chromosomes in prenatal diagnosis. Mol Cytogenet 2011, 4:2.

24. She X, Horvath JE, Jiang Z, Liu G, Furey TS, Christ L, Clark R, Graves T, Gulden CL, Alkan C, Bailey JA, Sahinalp C, Rocchi M, Haussler D, Wilson RK, Miller W, Schwartz S, Eichler EE: The structure and evolution of centromeric transition regions within the human genome. Nature 2004, 430:857-864.

25. Lengauer $C$, Green ED, Cremer T: Fluorescence in situ hybridization of YAC clones after Alu-PCR amplification. Genomics 1992, 13:826-828.

26. Liehr T, Heller A, Starke H, Claussen U: FISH banding methods: applications in research and diagnostics. Exp Rev Mol Diagn 2002, 2:217-225.

27. Weise A, Starke H, Heller A, Tönnies H, Volleth M, Stumm M, Senger G, Nietzel A, Claussen U, Liehr T: Chromosome 2 aberrations in clinical cases characterised by high resolution multicolour banding and region specific FISH probes. J Med Genet 2002, 39:434-439. 
28. Manvelyan M, Schreyer I, Höls-Herpertz I, Köhler S, Niemann R, Hehr U, Belitz B, Bartels I, Götz J, Huhle D, Kossakiewicz M, Tittelbach H, Neubauer S, Polityko A, Mazauric ML, Wegner R, Stumm M, Küpferling P, Süss F, Kunze H, Weise A, Liehr T, Mrasek K: Forty-eight new cases with infertility due to balanced chromosomal rearrangements: detailed molecular cytogenetic analysis of the 90 involved breakpoints. Int J Mol Med 2007, 19:855-864.

29. Liehr T, Karamysheva T, Merkas M, Brecevic L, Hamid AB, Ewers E, Mrasek K, Kosyakova N, Weise A: Somatic mosaicism in cases with small supernumerary marker chromosomes. Curr Genomics 2010, 11:432-439.

30. Weise A, Mrasek K, Fickelscher I, Claussen U, Cheung SW, Cai WW, Liehr T, Kosyakova N: Molecular definition of high-resolution multicolor banding probes: first within the human DNA sequence anchored FISH banding probe set. J Histochem Cytochem 2008, 56:487-493.

31. Christian SL, Fantes JA, Mewborn SK, Huang B, Ledbetter DH: Large genomic duplicons map to sites of instability in the prader-willi/angelman syndrome chromosome region (15q11-q13). Hum Mol Genet 1999, 8:1025-1037.

32. Torres-Juan L, Rosell J, Sanchez-de-la-Torre M, Fibla J, Heine-Suñer D: Analysis of meiotic recombination in 22q11.2, a region that frequently undergoes deletions and duplications. BMC Med Genet 2007, 8:14.

33. Li P, Pomianowski P, DiMaio MS, Florio JR, Rossi MR, Xiang B, Xu F, Yang $H$, Geng Q, Xie J, Mahoney MJ: Genomic characterization of prenatally detected chromosomal structural abnormalities using oligonucleotide array comparative genomic hybridization. Am J Med Genet A 2011, 155:1605-1615.

34. Slavin TP, Kuruvilla K, Curtis CA, Christ LA, Mitchell AL: Isolated skeletal malformations in a child with a small mosaic ring microduplication of 18 p11.21q11.2: Genotype-phenotype correlations. Am J Med Genet A 2011, 155:618-621.

35. Giardino D, Bettio D, Gottardi G, Rizzi N, Pierluigi M, Perfumo C, Cali A, Dagna Bricarelli F, Larizza L: FISH characterization of two supernumerary $\mathrm{r}(1)$ associated with distinct clinical phenotypes. Am J Med Genet 1999, 84:3770-3780.

36. Kosztolányi G, Brecevic L, Bajnòczky K, Schinzel A, Riegel M: Mosaic supernumerary ring chromosome 1 in a three-generational family: 10year follow-up report. Eur J Med Genet 2011, 54:152-156.

37. Liehr T, Nietzel A, Starke H, Heller A, Weise A, Kuechler A, Senger G, Ebner S, Martin T, Stumm M, Wegner R, Tönnies H, Hoppe C, Claussen U, Von Eggeling F: Characterization of small marker chromosomes (SMC) by recently developed molecular cytogenetic approaches. J Ass Genet Techn 2003, 29:5-10.

38. Tönnies $\mathrm{H}$, Hennies HC, Spohr HL, Neitzel H: Characterization of the first supernumerary tricentric ring chromosome 1 mosaicism by conventional and molecular cytogenetic techniques. Cytogenet Genome Res 2003, 103:28-33.

39. Rodriguez L, Starke H, Guardia NM, Tönnies H, Neitzel H, Kozlowski P, Mazauric ML, Heller A, Grondona FL, Mansilla E, Santos Muñoz MJ, Liehr T, Martínez-Frías ML: Three new cases with a supernumerary ring chromosome 1. Clin Dysmorphol 2005, 14:169-175.

40. Liehr T, Stumm M, Wegner RD, Bhatt S, Hickmann P, Patsalis PC, Meins M, Morlot S, Klaschka V, Ewers E, Hinreiner S, Mrasek K, Kosyakova N, Cai WW, Cheung SW, Weise A: 10p11.2 to $10 \mathrm{q} 11.2$ is a yet unreported region leading to unbalanced chromosomal abnormalities without phenotypic consequences. Cytogenet Genome Res 2009, 124:102-105.

41. Lichter P, Tang Ghang CJ, Call K, Hermanson G, Evans GA, Housman D, Ward DC: High resolution mapping of human chromosome 11 by in situ hybridization with cosmid clones. Science 1990, 247:64-69.

42. Lichter P, Cremer T: Chromosome analysis by non-isotopic in situ hybridization. In Human cytogenetics. A practical approach. Edited by Rooney DE, Czepulkowski BH. Oxford: IRL Press at Oxford University Press; 1992:157-192

doi:10.1186/1755-8166-6-45

Cite this article as: Castronovo et al:: Design and validation of a pericentromeric BAC clone set aimed at improving diagnosis and phenotype prediction of supernumerary marker chromosomes. Molecular Cytogenetics 2013 6:45.

\section{Submit your next manuscript to BioMed Central and take full advantage of:}

- Convenient online submission

- Thorough peer review

- No space constraints or color figure charges

- Immediate publication on acceptance

- Inclusion in PubMed, CAS, Scopus and Google Scholar

- Research which is freely available for redistribution

Submit your manuscript at www.biomedcentral.com/submit
C Biomed Central 